\title{
New Wilker-type and Huygens-type inequalities
}

\author{
Ling Zhu*1 (D), Branko Malešević ${ }^{2}$ (D) \\ ${ }^{1}$ Department of Mathematics, Zhejiang Gongshang University, Hangzhou City, Zhejiang Province, \\ 310018, China \\ ${ }^{2}$ School of Electrical Engineering, University of Belgrade, Bulevar kralja Aleksandra 73, 11000 Belgrade, \\ Serbia
}

\begin{abstract}
In this paper, we first determine the relationships between the first Wilker's inequality, the second Wilker's inequality, the first Huygens inequality, and the second Huygens inequality for circular functions and for hyperbolic functions, respectively. Then, we establish new Wilker-type inequalities and Huygens-type inequalities for two function pairs, $x / \sin ^{-1} x$ and $x / \tan ^{-1} x, x / \sinh ^{-1} x$ and $x / \tanh ^{-1} x$. Finally, we obtain some more general conclusions than the first work of this paper, which reveal the absolute monotonicity of four functions involving the four inequalities mentioned above.
\end{abstract}

Mathematics Subject Classification (2020). 26D15, 42A10

Keywords. Wilker-type inequalities, Huygens-type inequalities, circular functions, hyperbolic functions, inverse circular functions, inverse hyperbolic functions

\section{Introduction}

Let $0<x<\pi / 2$. Then

$$
\sin x<x<\tan x
$$

which can be rewrited as

or

$$
\begin{aligned}
& \frac{\sin x}{x}<1<\frac{\tan x}{x}, \\
& \frac{x}{\tan x}<1<\frac{x}{\sin x} .
\end{aligned}
$$

When the functions involved in (1.2) are taken into account in two forms of size relations, two famous inequalities called the first Wilker's inequality (see $[7,21,29,30,37,39]$ ), the first Huygens inequality (see $[3-5,8,9,11,28,32,43]$ ), it comes to the conclusions (1.4) and (1.5). The comparison of these two inequalities (see [6]) is shown as follows in (1.6).

$$
\begin{aligned}
\frac{1}{2}\left(\left(\frac{\sin x}{x}\right)^{2}+\frac{\tan x}{x}\right) & >1, \\
\frac{1}{3}\left(\frac{2 \sin x}{x}+\frac{\tan x}{x}\right) & >1,
\end{aligned}
$$

\footnotetext{
*Corresponding Author.

Email addresses: zhuling0571@163.com (L. Zhu), branko.malesevic@etf.rs (B. Malešević)

Received: 06.04.2019; Accepted: 26.04.2020
} 


$$
\frac{1}{2}\left(\left(\frac{\sin x}{x}\right)^{2}+\frac{\tan x}{x}\right)>\frac{1}{3}\left(\frac{2 \sin x}{x}+\frac{\tan x}{x}\right)>1 .
$$

Similar to (1.4) - (1.6), there are some conclusions (1.7) and (1.8) about the second Wilker's inequality (see $[23,24,32,43]$ ), the second Huygens inequality (see [23, 24]), and the comparison of the two inequalities as follows.

$$
\begin{aligned}
\frac{1}{2}\left(\left(\frac{x}{\sin x}\right)^{2}+\frac{x}{\tan x}\right) & >1, \\
\frac{1}{3}\left(\frac{2 x}{\sin x}+\frac{x}{\tan x}\right) & >1, \\
\frac{1}{2}\left(\left(\frac{x}{\sin x}\right)^{2}+\frac{x}{\tan x}\right) & >\frac{1}{3}\left(\frac{2 x}{\sin x}+\frac{x}{\tan x}\right)>1 .
\end{aligned}
$$

The last inequality chain is true due to

$$
\begin{aligned}
& \frac{1}{2}\left(\left(\frac{x}{\sin x}\right)^{2}+\frac{x}{\tan x}\right)-\frac{1}{3}\left(\frac{2 x}{\sin x}+\frac{x}{\tan x}\right) \\
= & \frac{1}{6}\left(2\left(\frac{x}{\sin x}\right)^{2}+\left(\frac{x}{\sin x}\right)^{2}+\frac{x}{\tan x}-4 \frac{x}{\sin x}\right) \\
> & \frac{1}{6}\left(2\left(\frac{x}{\sin x}\right)^{2}+2-4 \frac{x}{\sin x}\right)=\frac{1}{3}\left(1-\frac{x}{\sin x}\right)^{2}>0
\end{aligned}
$$

and (1.8). At the same time, we find that the inequality (1.7) plays a key role in the above derivation. Furthermore, the relationships between the first and second Wilker's inequality (see $[3,42]$ ), the first and second Huygens inequality (see [23, 24]) are given below.

$$
\begin{aligned}
\frac{1}{2}\left(\left(\frac{\sin x}{x}\right)^{2}+\frac{\tan x}{x}\right) & >\frac{1}{2}\left(\left(\frac{x}{\sin x}\right)^{2}+\frac{x}{\tan x}\right)>1, \\
\frac{1}{3}\left(\frac{2 \sin x}{x}+\frac{\tan x}{x}\right) & >\frac{1}{3}\left(\frac{2 x}{\sin x}+\frac{x}{\tan x}\right)>1 .
\end{aligned}
$$

The same case occurs in the hyperbolic functions (see [23, 24,36, 39, 41-44]).

Now let's turn to the discussion of similar inequalities for inverse circular functions. Let $0<x<1$. Then

$$
\tan ^{-1} x<x<\sin ^{-1} x
$$

which can be rewritten as

or

$$
\frac{\tan ^{-1} x}{x}<1<\frac{\sin ^{-1} x}{x},
$$

$$
\frac{x}{\sin ^{-1} x}<1<\frac{x}{\tan ^{-1} x} .
$$

Chen and Cheung [6] obtained an important conclusion about the inverse circular functions as follows.

$$
\left(\frac{x}{\sin ^{-1} x}\right)^{2}+\frac{x}{\tan ^{-1} x}<2,0<x<1 .
$$

Then, they used the arithmetic-geometric-harmonic mean inequality to prove the following inequality chain for $x \in(0,1)$ :

$$
\frac{1}{2}\left(\left(\frac{\sin ^{-1} x}{x}\right)^{2}+\frac{\tan ^{-1} x}{x}\right)>\frac{1}{3}\left(\frac{2 \sin ^{-1} x}{x}+\frac{\tan ^{-1} x}{x}\right)
$$




$$
\begin{aligned}
& >\left(\left(\frac{\sin ^{-1} x}{x}\right)^{2} \frac{\tan ^{-1} x}{x}\right)^{1 / 3} \\
& >\left(\frac{2}{1 /\left(\left(\sin ^{-1} x\right) / x\right)^{2}+1 /\left(\left(\tan ^{-1} x\right) / x\right)}\right)^{1 / 3}>1 .
\end{aligned}
$$

They established the inverse hyperbolic version of above results for $x \in(0,1)$ :

and

$$
\left(\frac{x}{\sinh ^{-1} x}\right)^{2}+\frac{x}{\tanh ^{-1} x}<2,
$$

$$
\begin{gathered}
\frac{1}{2}\left(\left(\frac{\sinh ^{-1} x}{x}\right)^{2}+\frac{\tanh ^{-1} x}{x}\right)>\frac{1}{3}\left(\frac{2 \sinh ^{-1} x}{x}+\frac{\tanh ^{-1} x}{x}\right) \\
>\left(\left(\frac{\sinh ^{-1} x}{x}\right)^{2} \frac{\tanh ^{-1} x}{x}\right)^{1 / 3} \\
>\left(\frac{2}{1 /\left(\left(\sinh ^{-1} x\right) / x\right)^{2}+1 /\left(\left(\tanh ^{-1} x\right) / x\right)}\right)^{1 / 3}>1 .
\end{gathered}
$$

The first task of this paper is to determine the relationship between the first Wilker's inequality, the second Wilker's inequality, the first Huygens inequality and the second Huygens inequality. The second one is to consider the results according to the form of the inequality (1.6) or (1.9) for two function pairs, $x / \sin ^{-1} x$ and $x / \tan ^{-1} x, x / \sinh ^{-1} x$ and $x / \tanh ^{-1} x$. Finally, we obtain some more general conclusions than the first work of this paper, which reveal the absolute monotonicity of four functions involving the above four inequalities.

\section{Main results}

This paper obtains the following main results.

Theorem 2.1. Let $x \in(0, \pi / 2)$. Then the inequality chain

$$
\begin{aligned}
& \frac{1}{2}\left(\left(\frac{\sin x}{x}\right)^{2}+\frac{\tan x}{x}\right)>\frac{1}{3}\left(\frac{2 \sin x}{x}+\frac{\tan x}{x}\right) \\
& >\frac{1}{2}\left(\left(\frac{x}{\sin x}\right)^{2}+\frac{x}{\tan x}\right)>\frac{1}{3}\left(\frac{2 x}{\sin x}+\frac{x}{\tan x}\right) \\
& >1 .
\end{aligned}
$$

holds.

Theorem 2.2. Let $x \in(0, \infty)$. Then the inequality chain

$$
\begin{aligned}
& \frac{1}{2}\left(\left(\frac{\sinh x}{x}\right)^{2}+\frac{\tanh x}{x}\right)>\frac{1}{3}\left(\frac{2 \sinh x}{x}+\frac{\tanh x}{x}\right) \\
& >\frac{1}{2}\left(\left(\frac{x}{\sinh x}\right)^{2}+\frac{x}{\tanh x}\right)>\frac{1}{3}\left(\frac{2 x}{\sinh x}+\frac{x}{\tanh x}\right) \\
& >1 .
\end{aligned}
$$

holds. 
Theorem 2.3. Let $x \in(0,1)$. Then the inequality chain

$$
\frac{1}{2}\left(\left(\frac{x}{\sin ^{-1} x}\right)^{2}+\frac{x}{\tan ^{-1} x}\right)<\frac{1}{3}\left(\frac{2 x}{\sin ^{-1} x}+\frac{x}{\tan ^{-1} x}\right)<1
$$

holds.

Theorem 2.4. Let $x \in(0,1)$. Then the inequality chain

$$
\frac{1}{2}\left(\left(\frac{x}{\sinh ^{-1} x}\right)^{2}+\frac{x}{\tanh ^{-1} x}\right)<\frac{1}{3}\left(\frac{2 x}{\sinh ^{-1} x}+\frac{x}{\tanh ^{-1} x}\right)<1
$$

holds.

Then we can obtain the following corollaries.

Corollary 2.5. Let $x \in(0,1)$. Then

$$
\begin{gathered}
\frac{1}{2}\left(\left(\frac{\sin ^{-1} x}{x}\right)^{2}+\frac{\tan ^{-1} x}{x}\right)>\frac{1}{3}\left(\frac{2 \sin ^{-1} x}{x}+\frac{\tan ^{-1} x}{x}\right)>1 \\
>\frac{1}{3}\left(\frac{2 x}{\sin ^{-1} x}+\frac{x}{\tan ^{-1} x}\right)>\frac{1}{2}\left(\left(\frac{x}{\sin ^{-1} x}\right)^{2}+\frac{x}{\tan ^{-1} x}\right) .
\end{gathered}
$$

Corollary 2.6. Let $x \in(0,1)$. Then

$$
\begin{aligned}
& \frac{1}{2}\left(\left(\frac{\sinh ^{-1} x}{x}\right)^{2}+\frac{\tanh ^{-1} x}{x}\right)>\frac{1}{3}\left(\frac{2 \sinh ^{-1} x}{x}+\frac{\tanh ^{-1} x}{x}\right)>1 \\
& >\frac{1}{3}\left(\frac{2 x}{\sinh ^{-1} x}+\frac{x}{\tanh ^{-1} x}\right)>\frac{1}{2}\left(\left(\frac{x}{\sinh ^{-1} x}\right)^{2}+\frac{x}{\tanh ^{-1} x}\right) .
\end{aligned}
$$

\section{Proofs}

\subsection{Proof of Theorem 2.1}

We shall complete the proof of Theorem 2.1 when proving second inequality of (2.1).

Computing directly gives

$$
\frac{1}{3}\left(\frac{2 \sin x}{x}+\frac{\tan x}{x}\right)-\frac{1}{2}\left(\left(\frac{x}{\sin x}\right)^{2}+\frac{x}{\tan x}\right)=\frac{\sin ^{2} x}{6 x \cos x} F(x),
$$

where

$$
\begin{aligned}
F(x) & =\frac{4 \cos x \sin ^{3} x+2 \sin ^{3} x-3 x^{3} \cos x-3 x^{2} \cos ^{2} x \sin x}{\sin ^{4} x} \\
& =4 \cot x+3 x^{2} \frac{1}{\sin x}-3 x^{2} \frac{1}{\sin ^{3} x}+2 \frac{1}{\sin x}+x^{3}\left(-3 \frac{\cos x}{\sin ^{4} x}\right) .
\end{aligned}
$$

Since

$$
\begin{aligned}
\left(\frac{1}{\sin x}\right)^{\prime} & =-\frac{\cos x}{\sin ^{2} x} \\
\left(\frac{1}{\sin x}\right)^{\prime \prime} & =\left(-\frac{\cos x}{\sin ^{2} x}\right)^{\prime}=\frac{2}{\sin ^{3} x}-\frac{1}{\sin x} \\
\left(\frac{1}{\sin ^{3} x}\right)^{\prime} & =-3 \frac{\cos x}{\sin ^{4} x},
\end{aligned}
$$

from

$$
\frac{1}{\sin x}=\frac{1}{x}+\sum_{n=1}^{\infty} \frac{2^{2 n}-2}{(2 n) !}\left|B_{2 n}\right| x^{2 n-1}, 0<|x|<\pi, \quad \text { (see [12]) }
$$


we have

$$
\begin{aligned}
\frac{1}{\sin ^{3} x}= & \frac{1}{2}\left(\left(\frac{1}{\sin x}\right)^{\prime \prime}+\frac{1}{\sin x}\right) \\
= & \frac{1}{2}\left(\frac{2}{x^{3}}+\sum_{n=2}^{\infty} \frac{\left(2^{2 n}-2\right)(2 n-1)(2 n-2)}{(2 n) !}\left|B_{2 n}\right| x^{2 n-3}\right) \\
& +\frac{1}{2}\left(\frac{1}{x}+\sum_{n=1}^{\infty} \frac{2^{2 n}-2}{(2 n) !}\left|B_{2 n}\right| x^{2 n-1}\right) \\
= & \frac{1}{x^{3}}+\sum_{n=2}^{\infty} \frac{\left(2^{2 n}-2\right)(2 n-1)(2 n-2)}{2(2 n) !}\left|B_{2 n}\right| x^{2 n-3} \\
& +\frac{1}{2 x}+\sum_{n=1}^{\infty} \frac{2^{2 n}-2}{2(2 n) !}\left|B_{2 n}\right| x^{2 n-1},
\end{aligned}
$$

and

$$
\begin{aligned}
-3 \frac{\cos x}{\sin ^{4} x}= & \frac{1}{2}\left(-\frac{6}{x^{4}}+\sum_{n=2}^{\infty} \frac{\left(2^{2 n}-2\right)(2 n-1)(2 n-2)(2 n-3)}{(2 n) !}\left|B_{2 n}\right| x^{2 n-4}\right) \\
& +\frac{1}{2}\left(-\frac{1}{x^{2}}+\sum_{n=1}^{\infty} \frac{\left(2^{2 n}-2\right)(2 n-1)}{(2 n) !}\left|B_{2 n}\right| x^{2 n-2}\right) \\
= & -\frac{3}{x^{4}}+\sum_{n=2}^{\infty} \frac{\left(2^{2 n}-2\right)(2 n-1)(2 n-2)(2 n-3)}{2(2 n) !}\left|B_{2 n}\right| x^{2 n-4} \\
& -\frac{1}{2 x^{2}}+\sum_{n=1}^{\infty} \frac{\left(2^{2 n}-2\right)(2 n-1)}{2(2 n) !}\left|B_{2 n}\right| x^{2 n-2} .
\end{aligned}
$$

We substitute the power series expansions of these functions into (3.2), and obtain

$$
\begin{aligned}
F(x)= & 4 \cot x+3 x^{2} \frac{1}{\sin x}+2 \frac{1}{\sin x}-3 x^{2} \frac{1}{\sin ^{3} x}+x^{3}\left(-3 \frac{\cos x}{\sin ^{4} x}\right) \\
= & 4\left(\frac{1}{x}-\sum_{n=1}^{\infty} \frac{2^{2 n}}{(2 n) !}\left|B_{2 n}\right| x^{2 n-1}\right)+3 x^{2}\left(\frac{1}{x}+\sum_{n=1}^{\infty} \frac{2^{2 n}-2}{(2 n) !}\left|B_{2 n}\right| x^{2 n-1}\right) \\
& +2\left(\frac{1}{x}+\sum_{n=1}^{\infty} \frac{2^{2 n}-2}{(2 n) !}\left|B_{2 n}\right| x^{2 n-1}\right) \\
& -3 x^{2}\left(\frac{1}{x^{3}}+\sum_{n=2}^{\infty} \frac{\left(2^{2 n}-2\right)(2 n-1)(2 n-2)}{2(2 n) !}\left|B_{2 n}\right| x^{2 n-3}\right) \\
& -3 x^{2}\left(\frac{1}{2 x}+\sum_{n=1}^{\infty} \frac{2^{2 n}-2}{2(2 n) !}\left|B_{2 n}\right| x^{2 n-1}\right) \\
& +x^{3}\left(-\frac{3}{x^{4}}+\sum_{n=2}^{\infty} \frac{\left(2^{2 n}-2\right)(2 n-1)(2 n-2)(2 n-3)}{2(2 n) !}\left|B_{2 n}\right| x^{2 n-4}\right) \\
& +x^{3}\left(-\frac{1}{2 x^{2}}+\sum_{n=1}^{\infty} \frac{\left(2^{2 n}-2\right)(2 n-1)}{2(2 n) !}\left|B_{2 n}\right| x^{2 n-2}\right) \\
= & \sum_{n=2}^{\infty} \frac{\left(2^{2 n}-2\right)(2 n-1)(2 n-2)(2 n-3)}{2(2 n) !}\left|B_{2 n}\right| x^{2 n-1} \\
& -3 \sum_{n=2}^{\infty} \frac{\left(2^{2 n}-2\right)(2 n-1)(2 n-2)}{2(2 n) !}\left|B_{2 n}\right| x^{2 n-1}
\end{aligned}
$$




$$
\begin{aligned}
& +2 \sum_{n=2}^{\infty} \frac{2^{2 n}-2}{(2 n) !}\left|B_{2 n}\right| x^{2 n-1}-4 \sum_{n=2}^{\infty} \frac{2^{2 n}}{(2 n) !}\left|B_{2 n}\right| x^{2 n-1} \\
& +3 \sum_{n=1}^{\infty} \frac{2^{2 n}-2}{(2 n) !}\left|B_{2 n}\right| x^{2 n+1}-3 \sum_{n=1}^{\infty} \frac{2^{2 n}-2}{2(2 n) !}\left|B_{2 n}\right| x^{2 n+1} \\
& +\sum_{n=1}^{\infty} \frac{\left(2^{2 n}-2\right)(2 n-1)}{2(2 n) !}\left|B_{2 n}\right| x^{2 n+1} \\
= & \sum_{n=2}^{\infty} \frac{\left(8 n^{3}-36 n^{2}+40 n-16\right) 2^{2 n}-16 n^{3}+72 n^{2}+16-80 n}{2(2 n) !}\left|B_{2 n}\right| x^{2 n-1} \\
& +\sum_{n=1}^{\infty} \frac{2(n+1)\left(2^{2 n}-2\right)}{2(2 n) !}\left|B_{2 n}\right| x^{2 n+1} \\
= & \sum_{n=2}^{\infty} 4 \frac{2^{2 n-1}\left(2 n^{3}+10 n-9 n^{2}-4\right)-\left(2 n^{3}+10 n-9 n^{2}-2\right)}{(2 n) !}\left|B_{2 n}\right| x^{2 n-1} \\
& +\sum_{n=2}^{\infty} \frac{n\left(2^{2 n-2}-2\right)}{(2 n-2) !}\left|B_{2 n-2}\right| x^{2 n-1} \\
:= & \sum_{n=2}^{\infty} a_{n} x^{2 n-1},
\end{aligned}
$$

where

$$
\begin{aligned}
a_{n}= & 4 \frac{2^{2 n-1}\left(2 n^{3}-9 n^{2}+10 n-4\right)-\left(2 n^{3}-9 n^{2}+10 n-2\right)}{(2 n) !}\left|B_{2 n}\right| \\
& +\frac{n\left(2^{2 n-2}-2\right)}{(2 n-2) !}\left|B_{2 n-2}\right|
\end{aligned}
$$

for $n \geq 2$.

Since

we first compute to obtain that

$$
\left|B_{2}\right|=\frac{1}{6},\left|B_{4}\right|=\frac{1}{30},\left|B_{6}\right|=\frac{1}{42},\left|B_{8}\right|=\frac{1}{30},
$$

$$
a_{2}=\frac{1}{6}, a_{3}=\frac{17}{315}, a_{4}=\frac{2509}{151200} .
$$

Then using mathematical induction we can prove

$$
2^{2 n-1}\left(2 n^{3}-9 n^{2}+10 n-4\right)-\left(2 n^{3}-9 n^{2}+10 n-2\right)>0
$$

or

$$
2^{2 n-1}>\frac{2 n^{3}-9 n^{2}+10 n-2}{2 n^{3}-9 n^{2}+10 n-4}
$$

for $n \geq 4$. In fact, when $n=4$, the inequality (3.5) holds. Now, we assume that the (3.5) holds for $n=m$. Then, in order to complete the proof of (3.5) is also true for $n=m+1$ it suffices to show that

$$
4 \frac{2 m^{3}-9 m^{2}+10 m-2}{2 m^{3}-9 m^{2}+10 m-4}>\frac{2(m+1)^{3}-9(m+1)^{2}+10(m+1)-2}{2(m+1)^{3}-9(m+1)^{2}+10(m+1)-4},
$$

which is true due to

$$
\begin{aligned}
& 4\left(2 m^{3}-9 m^{2}+10 m-2\right)\left(2(m+1)^{3}-9(m+1)^{2}+10(m+1)-4\right) \\
& -\left(2 m^{3}-9 m^{2}+10 m-4\right)\left(2(m+1)^{3}-9(m+1)^{2}+10(m+1)-2\right) \\
= & 12 m^{6}-72 m^{5}+129 m^{4}-54 m^{3}-3 m^{2}-42 m+12
\end{aligned}
$$




$$
\begin{aligned}
= & 12(m-4)^{6}+216(m-4)^{5}+1569(m-4)^{4}+5850(m-4)^{3} \\
& +11733(m-4)^{2}+11934(m-4)+4788 \\
> & 0 .
\end{aligned}
$$

So $a_{n}>0$ for $n \geq 2$. This leads to $F(x)>0$ for all $x \in(0, \pi / 2)$. The proof of (2.1) is complete via (3.1).

\subsection{Proof of Theorem 2.2}

Similarly, if we can prove second inequality of (2.2), we then complete the proof of Theorem 2.2.

Computing gives

$$
\frac{1}{3}\left(\frac{2 \sinh x}{x}+\frac{\tanh x}{x}\right)-\frac{1}{2}\left(\left(\frac{x}{\sinh x}\right)^{2}+\frac{x}{\tanh x}\right):=\frac{1}{24 x \cosh x \sinh ^{3} x} G(x),
$$

where

$$
\begin{aligned}
G(x)= & \cosh 4 x-3 \cosh 3 x-4 \cosh 2 x+\cosh 5 x+2 \cosh x \\
& -\frac{3}{2} x^{2} \cosh 4 x-6 x^{3} \sinh 2 x+\frac{3}{2} x^{2}+3 .
\end{aligned}
$$

Using the power series expansions of these hyperbolic functions, we have

$$
\begin{aligned}
G(x)= & \sum_{n=0}^{\infty} \frac{4^{2 n}}{(2 n) !} x^{2 n}-3 \sum_{n=0}^{\infty} \frac{3^{2 n}}{(2 n) !} x^{2 n}-4 \sum_{n=0}^{\infty} \frac{2^{2 n}}{(2 n) !} x^{2 n}+\sum_{n=0}^{\infty} \frac{5^{2 n}}{(2 n) !} x^{2 n} \\
& +2 \sum_{n=0}^{\infty} \frac{1}{(2 n) !} x^{2 n}-\frac{3}{2} x^{2} \sum_{n=0}^{\infty} \frac{4^{2 n}}{(2 n) !} x^{2 n}-6 x^{3} \sum_{n=0}^{\infty} \frac{2^{2 n+1}}{(2 n+1) !} x^{2 n+1} \\
& +\frac{3}{2} x^{2}+3 \\
= & \sum_{n=4}^{\infty} \frac{4^{2 n}-3 \cdot 3^{2 n}-4 \cdot 2^{2 n}+5^{2 n}+2}{(2 n) !} x^{2 n} \\
& -\frac{3}{2} \sum_{n=3}^{\infty} \frac{4^{2 n}}{(2 n) !} x^{2 n+2}-6 \sum_{n=2}^{\infty} \frac{2^{2 n+1}}{(2 n+1) !} x^{2 n+4} \\
= & \sum_{n=4}^{\infty} \frac{4^{2 n}-3 \cdot 3^{2 n}-4 \cdot 2^{2 n}+5^{2 n}+2}{(2 n) !} x^{2 n} \\
& -\sum_{n=4}^{\infty} \frac{3 \cdot 4^{2 n-2}}{2(2 n-2) !} x^{2 n}-\sum_{n=4}^{\infty} \frac{6 \cdot 2^{2 n-3}}{(2 n-3) !} x^{2 n} \\
:= & \sum_{n=4}^{\infty} \frac{1}{32(2 n) !} b_{n} x^{2 n},
\end{aligned}
$$

where

$$
\begin{aligned}
b_{n}= & 32 \cdot 5^{2 n}-\left(6 n^{2}-3 n-16\right) 2^{4 n+1}-32 \cdot 3^{2 n+1} \\
& -\left(6 n^{3}-9 n^{2}+3 n+4\right) 2^{2 n+5}+64
\end{aligned}
$$

for $n \geq 4$. We compute

$$
\begin{aligned}
c_{n}:= & b_{n+1}-25 b_{n} \\
= & 1536 \cdot 3^{2 n}+\left(108 n^{2}-438 n-384\right) 2^{4 n} \\
& +\left(126 n^{3}-261 n^{2}+63 n+84\right) 2^{2 n+5}-1536
\end{aligned}
$$


and obtain that

$$
\begin{aligned}
108 n^{2}-438 n-384 & >0, \\
\left(126 n^{3}-261 n^{2}+63 n+84\right) 2^{2 n+5}-1536 & >0
\end{aligned}
$$

hold for all $n \geq 5$. So $c_{n}>0$ for $n \geq 5$. This together with $c_{4}=17940480>0$ gives that $c_{n}>0$ for $n \geq 4$. Then via (3.8) we have $b_{n+1}>25 b_{n}$ holds for $n \geq 4$. This together with $b_{4}=860160>0$ gives that $b_{n}>0$ for $n \geq 4$. Then $G(x)>0$ for all $x \in(0, \pi / 2)$. The proof of $(2.2)$ is complete via (3.6).

\subsection{Proof of Theorem 2.3}

In order to prove Theorem 2.3 as simple as possible, we need a tool which offers a simple but efficient criterion to determine the sign of a kind of special power series, which we call as "sign rule of a kind of special power series".

Lemma 3.1 ([34], [33]). Let $\left\{a_{k}\right\}_{k=0}^{\infty}$ be a nonnegative real sequence with $a_{m}>0$ and $\sum_{k=m+1}^{\infty} a_{k}>0$ and let

$$
S(t)=-\sum_{k=0}^{m} a_{k} t^{k}+\sum_{k=m+1}^{\infty} a_{k} t^{k}
$$

be a convergent power series on the interval $(0, r)(r>0)$. (i) If $S\left(r^{-}\right) \leq 0$ then $S(t)<0$ for all $t \in(0, r)$. (ii) If $S\left(r^{-}\right)>0$ then there is the unique $t_{0} \in(0, r)$ such that $S(t)<0$ for $t \in\left(0, t_{0}\right)$ and $S(t)>0$ for $t \in\left(t_{0}, r\right)$.

(1) We first prove the left hand side of (2.3).

Let $\arcsin x=t$. Then the desired inequality is equivalent to

$$
\frac{1}{2}\left(\frac{\sin t}{t}\right)^{2}-\frac{2}{3} \frac{\sin t}{t}+\frac{1}{6} \frac{\sin t}{\arctan (\sin t)}=\frac{\sin t}{6}\left(\frac{1}{\arctan (\sin t)}-\frac{4 t-3 \sin t}{t^{2}}\right)<0,
$$

which is in turn equivalent to

$$
H(t):=\frac{t^{2}}{4 t-3 \sin t}-\arctan (\sin t)<0
$$

for $t \in(0, \pi / 2)$. Differentiation yields

$$
H^{\prime}(t)=\frac{\sin ^{3} t}{\left(1+\sin ^{2} t\right)(4 t-3 \sin t)^{2}} h(t),
$$

where

$$
h(t)=4 \frac{t^{2}}{\sin t}-6 \frac{t}{\sin ^{2} t}-9 \cot t-6 t+4 \frac{t^{2}}{\sin ^{3} t}+24 t \frac{\cos t}{\sin ^{2} t}+3 t^{2} \cot t-13 t^{2} \frac{\cos t}{\sin ^{3} t} .
$$

From

$$
\cot x=\frac{1}{x}-\sum_{n=1}^{\infty} \frac{2^{2 n}}{(2 n) !}\left|B_{2 n}\right| x^{2 n-1}, \quad 0<|x|<\pi, \quad \text { (see [10]) }
$$

and (3.3) we have

$$
\begin{aligned}
\frac{1}{\sin ^{2} t} & =-(\cot t)^{\prime}=\frac{1}{t^{2}}+\sum_{n=1}^{\infty} \frac{(2 n-1) 2^{2 n}}{(2 n) !}\left|B_{2 n}\right| t^{2 n-2} \\
\frac{\cos t}{\sin ^{2} t} & =-\left(\frac{1}{\sin t}\right)^{\prime}=\frac{1}{t^{2}}-\sum_{n=1}^{\infty} \frac{(2 n-1)\left(2^{2 n}-2\right)}{(2 n) !}\left|B_{2 n}\right| t^{2 n-2} \\
\frac{\cos t}{\sin ^{3} t} & =-\frac{1}{2}\left(\frac{1}{\sin ^{2} t}\right)^{\prime}=\frac{1}{t^{3}}-\sum_{n=2}^{\infty} \frac{(2 n-1)(n-1) 2^{2 n}}{(2 n) !}\left|B_{2 n}\right| t^{2 n-3}
\end{aligned}
$$

The above power series expansions and (3.4) give 


$$
\begin{aligned}
& h(t)=4 t+4 \sum_{n=1}^{\infty} \frac{2^{2 n}-2}{(2 n) !}\left|B_{2 n}\right| t^{2 n+1}-6 t\left(\frac{1}{t^{2}}+\sum_{n=1}^{\infty} \frac{(2 n-1) 2^{2 n}}{(2 n) !}\left|B_{2 n}\right| t^{2 n-2}\right) \\
& -9\left(\frac{1}{t}-\sum_{n=1}^{\infty} \frac{2^{2 n}}{(2 n) !}\left|B_{2 n}\right| t^{2 n-1}\right)+4 t^{2}\left(\frac{1}{2 t}+\frac{1}{2} \sum_{n=1}^{\infty} \frac{2^{2 n}-2}{(2 n) !}\left|B_{2 n}\right| t^{2 n-1}\right) \\
& +4 t^{2}\left(\frac{1}{t^{3}}+\frac{1}{2} \sum_{n=2}^{\infty} \frac{(2 n-1)(2 n-2)\left(2^{2 n}-2\right)}{(2 n) !}\left|B_{2 n}\right| t^{2 n-3}\right) \\
& +24 t\left(\frac{1}{t^{2}}-\sum_{n=1}^{\infty} \frac{(2 n-1)\left(2^{2 n}-2\right)}{(2 n) !}\left|B_{2 n}\right| t^{2 n-2}\right) \\
& +3 t^{2}\left(\frac{1}{t}-\sum_{n=1}^{\infty} \frac{2^{2 n}}{(2 n) !}\left|B_{2 n}\right| t^{2 n-1}\right) \\
& -13 t^{2}\left(\frac{1}{t^{3}}-\sum_{n=2}^{\infty} \frac{(2 n-1)(n-1) 2^{2 n}}{(2 n) !}\left|B_{2 n}\right| t^{2 n-3}\right)-6 t \\
& =4 t+4 \sum_{n=1}^{\infty} \frac{2^{2 n}-2}{(2 n) !}\left|B_{2 n}\right| t^{2 n+1}-\frac{6}{t}-6 \sum_{n=1}^{\infty} \frac{(2 n-1) 2^{2 n}}{(2 n) !}\left|B_{2 n}\right| t^{2 n-1} \\
& -\frac{9}{t}+9 \sum_{n=1}^{\infty} \frac{2^{2 n}}{(2 n) !}\left|B_{2 n}\right| t^{2 n-1}+4 t^{2}\left(\frac{1}{2 t}+\frac{1}{t^{3}}\right)+2 \sum_{n=1}^{\infty} \frac{2^{2 n}-2}{(2 n) !}\left|B_{2 n}\right| t^{2 n+1} \\
& +2 \sum_{n=2}^{\infty} \frac{(2 n-1)(2 n-2)\left(2^{2 n}-2\right)}{(2 n) !}\left|B_{2 n}\right| t^{2 n-1}+\frac{24}{t} \\
& -24 \sum_{n=1}^{\infty} \frac{(2 n-1)\left(2^{2 n}-2\right)}{(2 n) !}\left|B_{2 n}\right| t^{2 n-1}+3 t-3 \sum_{n=1}^{\infty} \frac{2^{2 n}}{(2 n) !}\left|B_{2 n}\right| t^{2 n+1} \\
& -\frac{13}{t}+13 \sum_{n=2}^{\infty} \frac{(2 n-1)(n-1) 2^{2 n}}{(2 n) !}\left|B_{2 n}\right| t^{2 n-1}-6 t \\
& =\sum_{n=1}^{\infty} \frac{3\left(2^{2 n}-4\right)}{(2 n) !}\left|B_{2 n}\right| t^{2 n+1} \\
& +\sum_{n=2}^{\infty} \frac{\left(34 n^{2}-111 n+56\right) 2^{2 n}-8(2 n-1)(n-7)}{(2 n) !}\left|B_{2 n}\right| t^{2 n-1} \\
& :=\sum_{n=2}^{\infty} \frac{k_{n}\left|B_{2 n-2}\right|+l_{n}\left|B_{2 n}\right|}{(2 n) !} t^{2 n-1}:=\sum_{n=2}^{\infty} p_{n} t^{2 n-1},
\end{aligned}
$$

where

$$
\begin{aligned}
k_{n} & =24 n(2 n-1)\left(2^{2 n-4}-1\right), \\
l_{n} & =\left(34 n^{2}-111 n+56\right) 2^{2 n}-8(2 n-1)(n-7) .
\end{aligned}
$$

A simple computation shows that $p_{2}=-1 / 2$. We claim that $p_{n}>0$ for $n \geq 3$. In fact, $k_{n}>0$ for $n \geq 3$. Also, since $\left(34 n^{2}-111 n+56\right)>0$, so for $n \geq 3$,

$$
l_{n}>\left(\left(34 n^{2}-111 n+56\right) 8-8(2 n-1)(n-7)\right)=8(32 n(n-3)+49)>0 .
$$

These indicate that $p_{n}>0$ for $n \geq 3$.

On the other hand, we see that

$$
h(\pi / 2)=2 \pi(\pi-3)>0 .
$$


By Lemma 3.1, there is a $t_{0} \in(0, \pi / 2)$ so that $h(t)<0$ for $t \in\left(0, t_{0}\right)$ and $h(t)>0$ for $t \in\left(t_{0}, \pi / 2\right)$, which in turn implies that $H(t)$ is decreasing on $\left(0, t_{0}\right)$ and increasing on $\left(t_{0}, \pi / 2\right)$. Consequently, we obtain

$$
\begin{aligned}
& H(t)<\lim _{t \rightarrow 0^{+}} H(t)=0 \text { for } t \in\left(0, t_{0}\right), \\
& H(t)<\lim _{t \rightarrow(\pi / 2)^{-}} H(t)=-\frac{1}{4} \frac{\pi(\pi-3)}{2 \pi-3}<0 \text { for } t \in\left(t_{0}, \pi / 2\right),
\end{aligned}
$$

that is, $H(t)<0$ for $t \in(0, \pi / 2)$. This completes the proof of the left hand side of $(2.3)$.

(2) We then prove the right hand side of (2.3).

The desired inequality is equivalent to

$$
2 \frac{x}{\sin ^{-1} x}+\frac{x}{\tan ^{-1} x}<3 .
$$

Since

we have

$$
\begin{aligned}
\frac{x}{\sin ^{-1} x} & <\frac{2+\sqrt{1-x^{2}}}{3}, \quad(\text { see }[15,16,21,38]) \\
\frac{x}{\tan ^{-1} x} & <1+\frac{1}{3} x^{2}, \quad(\text { see }[6])
\end{aligned}
$$

$$
2 \frac{x}{\sin ^{-1} x}+\frac{x}{\tan ^{-1} x}<\frac{2\left(2+\sqrt{1-x^{2}}\right)}{3}+1+\frac{1}{3} x^{2} .
$$

We can complete the proof of the right hand side of (2.3) as long as we can prove that

which is equivalent to $\left(1-\sqrt{1-x^{2}}\right)^{2}>0$.

$$
\frac{2\left(2+\sqrt{1-x^{2}}\right)}{3}+1+\frac{1}{3} x^{2}<3
$$

\subsection{Proof of Theorem 2.4}

(1) We first prove the left hand side of (2.4).

Since

$$
\begin{aligned}
& \frac{1}{3}\left(\frac{2 x}{\sinh ^{-1} x}+\frac{x}{\tanh ^{-1} x}\right)-\frac{1}{2}\left(\left(\frac{x}{\sinh ^{-1} x}\right)^{2}+\frac{x}{\tanh ^{-1} x}\right) \\
= & \frac{x}{6}\left(\frac{4}{\sinh ^{-1} x}-\frac{1}{\tanh ^{-1} x}-3 \frac{x}{\left(\sinh ^{-1} x\right)^{2}}\right),
\end{aligned}
$$

the desired inequality is equivalent to

$$
\tanh ^{-1} x>\frac{\left(\sinh ^{-1} x\right)^{2}}{4 \sinh ^{-1} x-3 x} .
$$

Let $\sinh ^{-1} x=t$. Then $x=\sinh t$, the above inequality is equivalent to

$$
\tanh ^{-1}(\sinh t)>\frac{t^{2}}{4 t-3 \sinh t} .
$$

Let

Then

$$
Q(t)=\tanh ^{-1}(\sinh t)-\frac{t^{2}}{4 t-3 \sinh t}
$$

$$
Q^{\prime}(t)=\frac{q(t)}{\left(1-\sinh ^{2} t\right)(4 t-3 \sinh t)^{2}}
$$


where

$$
\begin{aligned}
q(t)= & (\cosh t)(4 t-3 \sinh t)^{2}-\left(1-\sinh ^{2} t\right)\left(3 t^{2} \cosh t-6 t \sinh t+4 t^{2}\right) \\
= & \frac{9}{4} \cosh 3 t-\frac{9}{4} \cosh t+2 t^{2} \cosh 2 t+\frac{3}{4} t^{2} \cosh 3 t+\frac{21}{2} t \sinh t \\
& -12 t \sinh 2 t-\frac{3}{2} t \sinh 3 t+\frac{49}{4} t^{2} \cosh t-6 t^{2} .
\end{aligned}
$$

Expanding in power series of the hyperbolic functions leads to

$$
\begin{aligned}
q(t)= & \frac{9}{4} \sum_{n=0}^{\infty} \frac{(3 t)^{2 n}}{(2 n) !}-\frac{9}{4} \sum_{n=0}^{\infty} \frac{t^{2 n}}{(2 n) !}+2 t^{2} \sum_{n=0}^{\infty} \frac{(2 t)^{2 n}}{(2 n) !}+\frac{3}{4} t^{2} \sum_{n=0}^{\infty} \frac{(3 t)^{2 n}}{(2 n) !} \\
& +\frac{21}{2} t \sum_{n=0}^{\infty} \frac{t^{2 n+1}}{(2 n+1) !}-12 t \sum_{n=0}^{\infty} \frac{(2 t)^{2 n+1}}{(2 n+1) !}-\frac{3}{2} t \sum_{n=0}^{\infty} \frac{(3 t)^{2 n+1}}{(2 n+1) !} \\
& +\frac{49}{4} t^{2} \sum_{n=0}^{\infty} \frac{t^{2 n}}{(2 n) !}-6 t^{2} \\
= & \sum_{n=2}^{\infty} r_{n} t^{2 n+2},
\end{aligned}
$$

where

$$
r_{n}=\frac{4 n^{2}-6 n+17}{4(2 n+2) !} 3^{2 n+1}+\frac{2 n-11}{(2 n+1) !} 2^{2 n+1}+\frac{196 n^{2}+378 n+173}{4(2 n+2) !} .
$$

We find that

$$
r_{2}=\frac{1}{2}, r_{3}=\frac{11}{30}, r_{4}=\frac{411}{5600}, r_{5}=\frac{403}{50400},
$$

and $r_{n}>0$ for $n \geq 6$ due to $4 n^{2}-6 n+17>0$ and $2 n-11>0$. So $r_{n}>0$ for $n \geq 2$. This leads to that $q(t)>0$. Then $Q^{\prime}(t)>0$. So $Q(t)>Q\left(0^{+}\right)=0$, which completes the proof of the left hand side of (2.4).

(2) Then we prove the right hand side of (2.4).

The desired inequality is equivalent to

$$
2 \frac{x}{\sinh ^{-1} x}+\frac{x}{\tanh ^{-1} x}<3 .
$$

Since

we have

$$
\begin{aligned}
& \frac{x}{\sinh ^{-1} x}<\frac{2+\sqrt{x^{2}+1}}{3}, \quad(\text { see }[40]) \\
& \frac{x}{\tanh ^{-1} x}<\frac{1+2 \sqrt{1-x^{2}}}{3}, \quad \text { (see [6]) }
\end{aligned}
$$

$$
2 \frac{x}{\sinh ^{-1} x}+\frac{x}{\tanh ^{-1} x}<\frac{2\left(2+\sqrt{x^{2}+1}\right)}{3}+\frac{1+2 \sqrt{1-x^{2}}}{3} .
$$

In order to complete the proof of the right hand side of (2.4) it suffices to show

or

$$
\frac{2\left(2+\sqrt{x^{2}+1}\right)}{3}+\frac{1+2 \sqrt{1-x^{2}}}{3}<3
$$

$$
\begin{aligned}
& 2\left(2+\sqrt{x^{2}+1}\right)+1+2 \sqrt{1-x^{2}}<9 \\
& \Longleftrightarrow \sqrt{x^{2}+1}<2-\sqrt{1-x^{2}} \\
& \Longleftrightarrow x^{2}+1<4-4 \sqrt{1-x^{2}}+1-x^{2} \\
& \Longleftrightarrow x^{2}<2-2 \sqrt{1-x^{2}} .
\end{aligned}
$$


The last inequality is equivalent to $\left(1-\sqrt{1-x^{2}}\right)^{2}>0$.

\section{Further discussions}

Let us consider a real function $f:(a, b) \longrightarrow \mathbb{R}$ in case when exist finite limits $f^{(k)}(a+)=$ $\lim _{x \rightarrow a+} f^{(k)}(x)$ (for $k=0,1, \ldots, n$ and $n \in \mathbb{N}_{0}$ ) and $f(b-)=\lim _{x \rightarrow b-} f(x)$. We define

$$
\begin{aligned}
T_{n}^{f, a+}(x) & =\sum_{k=0}^{n} \frac{f^{(k)}(a+)}{k !}(x-a)^{k}, \\
R_{n}^{f, a+}(x) & =f(x)-T_{n}^{f, a+}(x),
\end{aligned}
$$

and

$$
\mathbb{T}_{n}^{f ; a+, b-}(x)=\left\{\begin{array}{cc}
T_{n-1}^{f, a+}(x)+\frac{1}{(b-a)^{n}} R_{n-1}^{f, a+}(b-)(x-a)^{n} & , \quad n \geq 1 \\
f(b-) & , \quad n=0
\end{array} .\right.
$$

Then the following statement is found to be true in [20, Theorem 3] and [18, Theorem 3].

Theorem 4.1. Let $f:(a, b) \longrightarrow \mathbb{R}$ be real analytic function with the power series:

$$
f(x)=\sum_{k=0}^{\infty} c_{k}(x-a)^{k},
$$

where $c_{k} \in \mathbb{R}$ and $c_{k} \geq 0$ for every $k \in \mathbb{N}_{0}$. Then,

$$
\begin{aligned}
T_{0}^{f, a+}(x) & \leq \ldots \leq T_{n}^{f, a+}(x) \leq T_{n+1}^{f, a+}(x) \leq \ldots \\
\ldots & \leq f(x) \leq \ldots \\
\ldots & \leq \mathbb{T}_{n+1}^{f ; a+, b-}(x) \leq \mathbb{T}_{n}^{f ; a+, b-}(x) \leq \ldots \leq \mathbb{T}_{0}^{f ; a+, b-}(x) .
\end{aligned}
$$

for every $x \in(a, b)$. If $c_{k} \in \mathbb{R}$ and $c_{k} \leq 0$ for every $k \in \mathbb{N}_{0}$, then the reversed inequality is true.

Let us emphasize that previous theorem improves result of Theorem 2 from [31]. Inspired by $[2,13,14,17,19,22,27]$, and [31], we obtain a conclusion more general than Theorem 2.1. The details are as follows.

Theorem 4.2. Let us form the functions

$$
\begin{aligned}
\varphi_{1}(x) & =\frac{1}{2}\left(\left(\frac{\sin x}{x}\right)^{2}+\frac{\tan x}{x}\right)-\frac{1}{3}\left(\frac{2 \sin x}{x}+\frac{\tan x}{x}\right):\left(0, \frac{\pi}{2}\right) \rightarrow R, \\
\varphi_{2}(x) & =\frac{1}{3}\left(\frac{2 \sin x}{x}+\frac{\tan x}{x}\right)-\frac{1}{2}\left(\left(\frac{x}{\sin x}\right)^{2}+\frac{x}{\tan x}\right):\left(0, \frac{\pi}{2}\right) \rightarrow R \\
\varphi_{3}(x) & =\frac{1}{2}\left(\left(\frac{x}{\sin x}\right)^{2}+\frac{x}{\tan x}\right)-\frac{1}{3}\left(\frac{2 x}{\sin x}+\frac{x}{\tan x}\right):\left(0, \frac{\pi}{2}\right) \rightarrow R, \\
\varphi_{4}(x) & =\frac{1}{3}\left(\frac{2 x}{\sin x}+\frac{x}{\tan x}\right)-1:\left(0, \frac{\pi}{2}\right) \longrightarrow R .
\end{aligned}
$$

Then functions $\varphi_{1}(x), \varphi_{2}(x), \varphi_{3}(x), \varphi_{4}(x)$ are real analytic with power series

$$
\varphi_{1}(x)=\sum_{k=2}^{\infty} s_{k}^{(1)} x^{2 k}, \varphi_{2}(x)=\sum_{k=2}^{\infty} s_{k}^{(2)} x^{2 k}, \varphi_{3}(x)=\sum_{k=2}^{\infty} s_{k}^{(3)} x^{2 k}, \varphi_{3}(x)=\sum_{k=2}^{\infty} s_{k}^{(3)} x^{2 k} .
$$

with positive coefficients

$$
s_{n}^{(1)}=\frac{1}{2} \frac{(-1)^{n} 2^{2 n+1}}{(2 n+2) !}+\frac{1}{6} \frac{\left(2^{2 n+2}-1\right) 2^{2 n+1}}{(2 n+2) !}\left|B_{2 n+2}\right|-\frac{2}{3} \frac{(-1)^{n}}{(2 n+1) !}>0,
$$




$$
\begin{aligned}
s_{n}^{(2)} & =\frac{2(-1)^{n}}{3(2 n+1) !}+\frac{\left(2^{2 n+2}-1\right) 2^{2 n+2}}{3(2 n+2) !}\left|B_{2 n+2}\right|-\frac{(n-1) 2^{2 n}}{(2 n) !}\left|B_{2 n}\right|>0, \\
s_{n}^{(3)} & =\frac{(3 n-4) 2^{2 n}+4}{3(2 n) !}\left|B_{2 n}\right|>0, \\
s_{n}^{(4)} & =\frac{2^{2 n}-4}{3(2 n) !}\left|B_{2 n}\right|>0
\end{aligned}
$$

for $n=2,3, \ldots$ Let it be that $j \in\{1,2,3,4\}$ and $c \in(0, \pi / 2)$ fixed. Then the double inequality

$$
\begin{aligned}
0<T_{2}^{\varphi_{j}, 0+}{ }^{(x)} & \leq T_{3}^{\varphi_{j}, 0+}(x) \ldots \leq T_{n}^{\varphi_{j}, 0+}(x) \leq T_{n+1}^{\varphi_{j}, 0+}(x) \leq \ldots \\
\ldots & \leq \varphi_{j}(x) \leq \ldots \\
\ldots & \leq \mathbb{T}_{n+1}^{\varphi_{j} ; 0+, c-}(x) \leq \mathbb{T}_{n}^{\varphi_{j} ; 0+, c-}(x) \leq \ldots \mathbb{T}_{3}^{\varphi_{j} ; 0+, c-}(x) \leq \mathbb{T}_{2}^{\varphi_{j} ; 0+, c-}
\end{aligned}
$$

holds for all $x \in(0, c)$.

Proof. For example, let us consider only case $j=2$. Since

$$
\varphi(x)=\varphi_{2}(x)=\frac{1}{3}\left(\frac{2 \sin x}{x}+\frac{\tan x}{x}\right)-\frac{1}{2}\left(\left(\frac{x}{\sin x}\right)^{2}+\frac{x}{\tan x}\right)=\sum_{k=2}^{\infty} s_{k} x^{2 k},
$$

where

$$
s_{n}=s_{n}^{(2)}=\frac{2(-1)^{n}}{3(2 n+1) !}+\frac{\left(2^{2 n+2}-1\right) 2^{2 n+2}}{3(2 n+2) !}\left|B_{2 n+2}\right|-\frac{(n-1) 2^{2 n}}{(2 n) !}\left|B_{2 n}\right|, \quad n \geq 2 .
$$

We can prove $s_{n}>0$ holds for all $n \geq 2$. In [10,1.3.1.4] or [46, 1.3.10], we can find the following power series expansion:

$$
\tan x=\sum_{n=1}^{\infty} \frac{2^{2 n}-1}{(2 n) !} 2^{2 n}\left|B_{2 n}\right| x^{2 n-1},|x|<\frac{\pi}{2} .
$$

Based on (3.9), (3.10), and (4.7) follows

$$
\begin{aligned}
\varphi(x)= & \frac{2}{3} \frac{\sin x}{x}+\frac{1}{3} \frac{\tan x}{x}-\frac{1}{2}\left(\frac{x}{\sin x}\right)^{2}-\frac{1}{2} \frac{x}{\tan x} \\
= & \frac{2}{3} \sum_{n=0}^{\infty} \frac{(-1)^{n}}{(2 n+1) !} x^{2 n}+\frac{1}{3} \sum_{n=1}^{\infty} \frac{2^{2 n}-1}{(2 n) !} 2^{2 n}\left|B_{2 n}\right| x^{2 n-2} \\
& -\frac{1}{2}\left[1+\sum_{n=1}^{\infty} \frac{2^{2 n}(2 n-1)}{(2 n) !}\left|B_{2 n}\right| x^{2 n}\right]-\frac{1}{2}\left[1-\sum_{n=1}^{\infty} \frac{2^{2 n}}{(2 n) !}\left|B_{2 n}\right| x^{2 n}\right] \\
= & \frac{2}{3} \sum_{n=0}^{\infty} \frac{(-1)^{n}}{(2 n+1) !} x^{2 n}+\frac{1}{3} \sum_{n=0}^{\infty} \frac{2^{2 n+2}-1}{(2 n+2) !} 2^{2 n+2}\left|B_{2 n+2}\right| x^{2 n} \\
& -\frac{1}{2}\left[1+\sum_{n=1}^{\infty} \frac{2^{2 n}(2 n-1)}{(2 n) !}\left|B_{2 n}\right| x^{2 n}\right]-\frac{1}{2}\left[1-\sum_{n=1}^{\infty} \frac{2^{2 n}}{(2 n) !}\left|B_{2 n}\right| x^{2 n}\right] \\
= & \sum_{n=2}^{\infty} \frac{2(-1)^{n}}{3(2 n+1) !} x^{2 n}+\sum_{n=2}^{\infty} \frac{\left(2^{2 n+2}-1\right) 2^{2 n+2}}{3(2 n+2) !}\left|B_{2 n+2}\right| x^{2 n} \\
& -\sum_{n=2}^{\infty} \frac{2^{2 n-1}(2 n-1)}{(2 n) !}\left|B_{2 n}\right| x^{2 n}+\sum_{n=2}^{\infty} \frac{2^{2 n-1}}{(2 n) !}\left|B_{2 n}\right| x^{2 n} \\
= & \sum_{n=2}^{\infty} \frac{2(-1)^{n}}{3(2 n+1) !} x^{2 n}+\sum_{n=2}^{\infty} \frac{\left(2^{2 n+2}-1\right) 2^{2 n+2}}{3(2 n+2) !}\left|B_{2 n+2}\right| x^{2 n}-\sum_{n=2}^{\infty} \frac{(n-1) 2^{2 n}}{(2 n) !}\left|B_{2 n}\right| x^{2 n}
\end{aligned}
$$




$$
=\sum_{n=2}^{\infty} s_{n} x^{2 n}
$$

Next, we shall prove that $s_{n}>0$ for all $n \geq 2$.

(i) When $n$ is even,

$$
s_{n}=\frac{2}{3(2 n+1) !}+\frac{\left(2^{2 n+2}-1\right) 2^{2 n+2}}{3(2 n+2) !}\left|B_{2 n+2}\right|-\frac{(n-1) 2^{2 n}}{(2 n) !}\left|B_{2 n}\right|,
$$

we complete the proof of $s_{n}>0$ as long as

$$
\frac{\left(2^{2 n+2}-1\right) 2^{2 n+2}}{3(2 n+2) !}\left|B_{2 n+2}\right|-\frac{(n-1) 2^{2 n}}{(2 n) !}\left|B_{2 n}\right|>0,
$$

or

$$
\frac{\left|B_{2 n+2}\right|}{\left|B_{2 n}\right|}>\frac{\frac{(n-1) 2^{2 n}}{(2 n) !}}{\frac{\left(2^{2 n+2}-1\right) 2^{2 n+2}}{3(2 n+2) !}}=\frac{(n-1) 2^{2 n}}{(2 n) !} \frac{3(2 n+2) !}{\left(2^{2 n+2}-1\right) 2^{2 n+2}} .
$$

Since

$$
\frac{\left|B_{2 n+2}\right|}{\left|B_{2 n}\right|}>\frac{2^{2 n-1}-1}{2^{2 n+1}-1} \frac{(2 n+2)(2 n+1)}{\pi^{2}},(\text { see }[1,25,26,35,45])
$$

we complete the proof when proving

$$
\frac{2^{2 n-1}-1}{2^{2 n+1}-1} \frac{(2 n+2)(2 n+1)}{\pi^{2}}>\frac{(n-1) 2^{2 n}}{(2 n) !} \frac{3(2 n+2) !}{\left(2^{2 n+2}-1\right) 2^{2 n+2}},
$$

that is,

$$
2^{2 n}>\frac{6\left[\pi^{2}(n-1)+3\right]}{8} \text { for } n \geq 2 .
$$

It is not difficult to prove the above formula by mathematical induction.

(ii) When $n$ is odd,

$$
s_{n}=-\frac{2}{3(2 n+1) !}+\frac{\left(2^{2 n+2}-1\right) 2^{2 n+2}}{3(2 n+2) !}\left|B_{2 n+2}\right|-\frac{(n-1) 2^{2 n}}{(2 n) !}\left|B_{2 n}\right| .
$$

By

we have

$$
\frac{2(2 n) !}{(2 \pi)^{2 n}} \frac{1}{1-2^{-2 n}}<\left|B_{2 n}\right|<\frac{2(2 n) !}{(2 \pi)^{2 n}} \frac{1}{1-2^{1-2 n}}, n=1,2, \cdots, \quad \text { (see [1]) }
$$

$$
\begin{aligned}
s_{n}> & -\frac{2}{3(2 n+1) !}+\frac{\left(2^{2 n+2}-1\right) 2^{2 n+2}}{3(2 n+2) !} \frac{2(2 n+2) !}{(2 \pi)^{2 n+2}} \frac{1}{1-2^{-2 n-2}} \\
& -\frac{(n-1) 2^{2 n}}{(2 n) !} \frac{2(2 n) !}{(2 \pi)^{2 n}} \frac{1}{1-2^{1-2 n}} \\
= & -\frac{2}{3(2 n+1) !}+\frac{\left(2^{2 n+2}-1\right) 2^{2 n+2}}{3(2 n+2) !} \frac{2(2 n+2) !}{(2 \pi)^{2 n+2}} \frac{2^{2 n+2}}{2^{2 n+2}-1} \\
& -\frac{(n-1) 2^{2 n}}{(2 n) !} \frac{2(2 n) !}{(2 \pi)^{2 n}} \frac{2^{2 n-1}}{2^{2 n-1}-1} \\
= & \frac{2 \cdot 2^{2 n+2}}{3 \pi^{2 n+2}}-\frac{2^{2 n}(n-1)}{\left(2^{2 n-1}-1\right) \pi^{2 n}}-\frac{2}{3(2 n+1) !} .
\end{aligned}
$$

Since

$$
s_{n} \quad>\quad 0 \Longleftrightarrow \frac{2}{3} \frac{\left(4 \cdot 2^{2 n}-3 \pi^{2} n+3 \pi^{2}-8\right) 2^{2 n}}{\pi^{2 n} \pi^{2}\left(2^{2 n}-2\right)}>\frac{2}{3(2 n+1) !}
$$




$$
\begin{aligned}
& \Longleftrightarrow \frac{\left(4 \cdot 2^{2 n}-3 \pi^{2} n+3 \pi^{2}-8\right) 2^{2 n}}{\pi^{2 n+2}\left(2^{2 n}-2\right)}>\frac{1}{(2 n+1) !} \\
& \Longleftrightarrow\left(4 \cdot 2^{2 n}-3 \pi^{2} n+3 \pi^{2}-8\right) 2^{2 n}(2 n+1) !>\pi^{2 n+2}\left(2^{2 n}-2\right),
\end{aligned}
$$

and

we have

$$
n !>\left(\frac{n}{3}\right)^{n}, n \in \mathbb{N}
$$

$$
(2 n+1) !>\left(\frac{2 n+1}{3}\right)^{2 n+1}>2^{2 n+1}, n \in \mathbb{N}_{0},
$$

$$
\left(4 \cdot 2^{2 n}-3 \pi^{2} n+3 \pi^{2}-8\right) 2^{2 n}(2 n+1) !>\left(4 \cdot 2^{2 n}-3 \pi^{2} n+3 \pi^{2}-8\right) 2^{2 n} 2^{2 n+1} .
$$

Then we complete the proof when proving

$$
\left(4 \cdot 2^{2 n}-3 \pi^{2} n+3 \pi^{2}-8\right) 2^{2 n} 2^{2 n+1}>\pi^{2 n+2}\left(2^{2 n}-2\right),
$$

that is,

$$
\begin{aligned}
t_{n} & =\left(4 \cdot 2^{2 n}-3 \pi^{2} n+3 \pi^{2}-8\right) 2^{2 n} 2^{2 n+1}-\pi^{2 n+2}\left(2^{2 n}-2\right) \\
& =8 \cdot 8^{2 n}-(2 \pi)^{2 n} \pi^{2}-2 \cdot 4^{2 n}\left[3 \pi^{2}(n-1)+8\right]+2 \pi^{2} \pi^{2 n} \\
& >0
\end{aligned}
$$

for all $n \geq 2$. We find

$$
t_{2}=28672-14 \pi^{6}-1536 \pi^{2}=52.839 \ldots>0,
$$

and

$$
\begin{aligned}
t_{n+1}-64 t_{n}= & {\left[4 \pi^{2}(4-\pi)(\pi+4) 2^{2 n}-\left(128 \pi^{2}-2 \pi^{4}\right)\right] \pi^{2 n} } \\
& +96 \cdot 4^{2 n}\left(3 \pi^{2} n-4 \pi^{2}+8\right) \\
> & 0 .
\end{aligned}
$$

Then $t_{n}>0$ for all $n \geq 2$.

Remark 4.3. Obviously, Theorem 2.1 is a simple corollary of Theorem 4.2.

Acknowledgment. The authors are grateful to anonymous referees for their careful corrections to and valuable comments on the original version of this paper.

The first author was supported by the National Natural Science Foundation of China (no. 61772025). The second author was supported in part by the Serbian Ministry of Education, Science and Technological Development, under projects ON 174032 and III 44006.

\section{References}

[1] M. Abramowitz and I. A. Stegun (Eds), Handbook of Mathematical Functions with Formulas, Graphs, and Mathematical Tables, National Bureau of Standards, Applied Mathematics Series 55, Dover Publications, 1972.

[2] B. Banjac, M. Makragić, and B. Malešević, Some notes on a method for proving inequalities by computer, Results Math. 69, 161-176, 2016.

[3] A. Baricz and J. Sandor, Extensions of the generalized Wilker inequality to Bessel functions, J. Math. Inequal. 2 (3), 397-406, 2008.

[4] F. Cajori, A History of Mathematics, 2nd ed., New York, 1929.

[5] F.T. Campan, The Story of Number $\pi$, Ed. Albatros, Romania, 1977. 
[6] C.-P. Chen and W.-S. Cheung, Wilker- and Huygens-type inequalities and solution to Oppenheim's problem, Int. Trans. Spec. Funct. 23 (5), 325-336, 2012.

[7] B.-N. Guo, B.-M. Qiao, F. Qi, and W. Li, On new proofs of Wilker inequalities involving trigonometric functions, Math. Inequal. Appl. 6 (1), 19-22, 2003.

[8] C. Huygens, Oeuvres Completes, Publiees Par la Societe Hollandaise des Science, Haga, 20 volumes, 1888-1940.

[9] A.P. Iuskevici, History of Mathematics in 16th and 17th Centuries, Moskva, 1961.

[10] A. Jeffrey, Handbook of Mathematical Formulas and Integrals, 3rd ed., Elsevier Acad. Press, San Diego, CA, 2004.

[11] J.-C. Kuang, Applied Inequalities, 3rd ed. (in Chinese), Shangdong Science and Technology Press, Jinan City, Shangdong Province, China, 2004.

[12] J.-L. Li, An identity related to Jordan's inequality, Int. J. Math. Math. Sci. 6, Article ID $76782,2016$.

[13] T. Lutovac, B. Malešević, and C. Mortici, The natural algorithmic approach of mixed trigonometric-polynomial problems, J. Inequal. Appl. 2017, Article No: 116, 2017.

[14] T. Lutovac, B. Malešević, and M. Rašajski, A new method for proving some inequalities related to several special functions, Results Math. 73, Article No: 100, 2018.

[15] B.J. Malešević, Application of $\lambda$-method on Shafer-Fink's inequality, Univ. Beograd. Publ. Elektrotehn. Fak. Ser. Mat. 8, 103-105, 1997.

[16] B.J. Malešević, An application of $\lambda$-method on inequalities of Shafer-Fink's type, Math. Inequal. Appl. 10, 529-534, 2007.

[17] B. Malešević, T. Lutovac, M. Rašajski, and C. Mortici, Extensions of the natural approach to refinements and generalizations of some trigonometric inequalities, Adv. Difference Equ. 2018, Article No: 90, 2018.

[18] B. Malešević, M. Nenezić, L. Zhu, B. Banjac and M. Petrovic, Some new estimates of precision of Cusa-Huygens and Huygens approximations, accepted in Appl. Anal. Discrete Math., 2020.

[19] B. Malešević, M. Rašajski, and T. Lutovac, Refinements and generalizations of some inequalities of Shafer-Fink's type for the inverse sine function, J. Inequal. Appl. 2017, Article No: 275, 2017.

[20] B. Malešević, M. Rašajski, and T. Lutovac, Double-sided Taylor's approximations and their applications in Theory of analytic inequalities. in: Differential and Integral Inequalities, Th.M. Rassias, D. Andrica (eds.), Optimization and Its Applications, vol. 151, 569-582, 2019.

[21] D.S. Mitrinović, Analytic Inequalities, Springer-Verlag, New York, Berlin 1970.

[22] M. Nenezić and L. Zhu, Some improvements of Jordan-Steckin and Becker-Stark inequalities, Appl. Anal. Discrete Math. 12, 244-256, 2018.

[23] E. Neuman, Wilker and Huygens-type inequalities for Jacobian elliptic and theta functions, Int. Trans. Spec. Funct. 25 (3), 240-248, 2014.

[24] E. Neuman and J. Sandor, On some inequalities involving trigonometric and hyperbolic functions with emphasis on the Cusa-Huygens, Wilker, and Huygens inequalities, Math. Inequal. Appl. 13 (4), 715-723, 2010.

[25] F. Qi, Notes on a double inequality for ratios of any two neighbouring non-zero Bernoulli numbers, Turkish J. Anal. Number Theory 6 (5), 129-131, 2018.

[26] F. Qi, A double inequality for the ratio of two non-zero neighbouring Bernoulli numbers, J. Comput. Appl. Math. 351, 1-5, 2019.

[27] M. Rašajski, T. Lutovac, and B. Malešević, About some exponential inequalities related to the sinc function, J. Inequal. Appl. 2018, Article No: 150, 2018.

[28] J. Sandor and M. Bencze, On Huygens's trigonometric inequality, RGMIA Research Report Collection 8 (3), Art. 14, 2005.

[29] J.S. Sumner, A.A. Jagers, M. Vowe, and J. Anglesio, Inequalities involving trigonometric functions, Amer. Math. Monthly 98, 264-267, 1991. 
[30] J.B. Wilker, Problem E 3306, Amer. Math. Monthly 96, 55, 1989.

[31] S.-H. Wu, L. Debnath, A generalization of L'Hôspital-type rules for monotonicity and its application, Appl. Math. Lett. 22 (2), 284-290, 2009.

[32] S.-H. Wu, H.M. Srivastava, A weighted and exponential generalization of Wilker's inequality and its applications, Int. Trans. Spec. Funct. 18 (8), 529-535, 2008.

[33] Z.-H. Yang, W.-M. Qian, Y.-M. Chu and W. Zhang, On approximating the arithmeticgeometric mean and complete elliptic integral of the first kind, J. Math. Anal. Appl. 462, 1714-1726, 2018.

[34] Z.-H. Yang and J.-F. Tian, Convexity and monotonicity for the elliptic integrals of the first kind and applications, Appl. Anal. Discrete Math. 13 (1), 240-260, 2019.

[35] Z.-H. Yang and J.-F. Tian, Sharp bounds for the ratio of two zeta functions, J. Comput. Appl. Math. 364, 112359, 14 pages, 2020.

[36] L. Zhu, On Shafer-Fink inequalities, Math. Inequal. Appl. 8 (4), 571-574, 2005.

[37] L. Zhu, A new simple proof of Wilker's inequality, Math. Inequal. Appl. 8 (4), 749 $750,2005$.

[38] L. Zhu, A solution of a problem of Oppenheim, Math. Inequal. Appl. 10, 57-61, 2007.

[39] L. Zhu, On Wilker-type inequalities, Math. Inequal. Appl. 10 (4), 727-731, 2007.

[40] L. Zhu, New inequalities of Shafer-Fink type for arc hyperbolic sine, J. Inequal. Appl. 2008, Article ID 368275, 2008.

[41] L. Zhu, Some new inequalities of the Huygens type, Comput. Math. Appl. 58, 11801182, 2009.

[42] L. Zhu, Some new Wilker-type inequalities for circular and hyperbolic functions, Abstr. Appl. Anal. 2009, Article ID 485842, 9 pages, 2009.

[43] L. Zhu, A source of inequalities for circular functions, Comput. Math. Appl. 58, 1998-2004, 2009.

[44] L. Zhu, Inequalities for hyperbolic functions and their applications, J. Inequal. Appl. 2010, Article ID 130821, 10 pages, 2010.

[45] L. Zhu, New bounds for the ratio of two adjacent even-indexed Bernoulli numbers, Rev. R. Acad. Cienc. Exactas Fís. Nat. Ser. A Mat. 114, 2020.

[46] D. Zwillinger, CRC Standard Mathematical Tables and Formulae, CRC Press, 1996. 